\title{
TOROIDAL SURGERIES ON HYPERBOLIC KNOTS, II *
}

\author{
MASAKAZU TERAGAITO ${ }^{\dagger}$
}

\begin{abstract}
For a hyperbolic knot $K$ in $S^{3}$, a toroidal surgery on $K$ is integral or half-integral. In the previous paper, we proved that all integers occur among the toroidal slopes of hyperbolic knots. Hence there is no universal upper bound for toroidal slopes, generally. We propose an upper bound in terms of genera of knots, and we show that this is the case for two special but important classes, i.e., alternating knots and genus one knots.
\end{abstract}

1. Introduction. Let $K$ be a knot in the 3-sphere $S^{3}$, and let $E(K)=$ $S^{3}-\operatorname{Int} N(K)$ be its exterior. A slope on $\partial E(K)$ is the isotopy class of an essential unoriented simple loop. As usual [20], the set of slopes on $\partial E(K)$ is parameterized by $\mathbb{Q} \cup\{\infty\}$. For a slope $r$ on $\partial E(K), K(r)$ denotes the closed orientable 3-manifold obtained by $r$-Dehn surgery on $K$.

For a hyperbolic knot $K, K(r)$ is hyperbolic for all but finitely many $r$ [24], which are referred to as exceptional slopes. A closed 3-manifold is toroidal if it contains an incompressible torus. If $K(r)$ is toroidal, the surgery (or the slope) is said to be toroidal. An exceptional slope is conjectured to yield either a toroidal manifold or a Seifert fibered manifold.

Gordon and Luecke [14] showed that if $K(m / n)$ is toroidal then $|n| \leq 2$. In other words, a toroidal slope on a hyperbolic knot is either an integer or a half-integer. In the previous paper [23], we showed that every integer can be a toroidal slope for some hyperbolic knot. Thus there is no universal upper bound for toroidal slopes, but Ichihara [18] recently showed that a toroidal slope $r$ on a hyperbolic knot $K$ satisfies the inequality $|r|<3 \cdot 2^{7 / 4} g(K)$, where $g(K)$ denotes the genus of $K$.

By an inspection of the known examples, we propose the following conjecture:

CONJECTURE 1.1. If a hyperbolic knot $K$ in $S^{3}$ has a toroidal slope $r$, then $|r| \leq 4 g(K)$.

In this paper, we prove this conjecture for two special but important classes of hyperbolic knots, i.e., alternating knots and genus one knots. In fact, we prove slightly stronger conclusions in both cases.

THEOREM 1.2. Let $K$ be a genus one hyperbolic knot. If $r$ is toroidal, then $|r|=0,1,2$ or 4 . Furthermore, if $|r|=2$ or 4 , then $K(r)$ contains an incompressible torus meeting the attached solid torus in two meridian disks. Also, if $|r|=4$, then $K$ is a twist knot and it bounds a once-punctured Klein bottle whose boundary slope is $r$.

In fact, the slopes 0,2 and 4 can be realized. For example, 0 and 4 are toroidal slopes for the figure-eight knot, and 2 is such one for $9_{46}$ in the knot table $[6,17]$. But we do not know whether 1 can be realized or not.

THEOREM 1.3. Let $K$ be an alternating hyperbolic knot. If $r$ is toroidal, then $|r| \leq 4 g(K)$. Furthermore, if the equality holds, then $K$ bounds a once-punctured Klein bottle whose boundary slope is $r$.

*Received September 17, 2002; accepted for publication February 27, 2003.

†Department of Mathematics and Mathematics Education, Graduate School of Education, Hiroshima University, 1-1-1 Kagamiyama, Higashi-hiroshima 739-8524, Japan (teragai@hiroshimau.ac.jp). 
In fact, there are examples of alternating hyperbolic knots showing this bound is best possible for each genus. The simplest one is the figure-eight knot again. It bounds a once-punctured Klein bottle whose boundary slope is \pm 4 . See Section 3 .

The author would like to thank the referee for helpful comments.

2. Genus one case. In this section, we prove Theorem 1.2.

Let $K$ be a hyperbolic knot of genus one in $S^{3}$, and let $S$ be a minimal genus Seifert surface of $K$ properly embedded in $E(K)$. Suppose that $K(r)$ is toroidal for a slope $r$.

LEMMA 2.1. $r$ is an integer.

Proof. If not, then $r$ is a half-integer by [14]. Then $K$ is one of Eudave-Muñoz's knots [16], and in particular, $K$ has tunnel number one (see [13]). Scharlemann [21] showed that a genus one, tunnel number one, hyperbolic knot is 2-bridge. Therefore $K$ is 2-bridge. This contradicts the fact that 2-bridge knots admit only integral toroidal surgeries $[1,3]$. $\mathrm{C}$

It is known that 0 is toroidal [8]. For our purpose, we may assume that $r>0$.

LEMMA 2.2. $r \leq 5$.

Proof. By [12], the distance between two toroidal slopes is at most 5 except four specific manifolds. Among those four manifolds, the figure-eight knot exterior is the only case of knot exteriors in $S^{3}$. But it has exactly three toroidal slopes $-4,0,4$. Thus we have $r \leq 5$. $\square$

Let $\widehat{T}$ be an incompressible torus in $K(r)$. Since $r>0, \widehat{T}$ is separating. We can assume that $\widehat{T}$ intersects the attached solid torus $V$ in a disjoint union of meridian disks. Let $|\widehat{T} \cap V|=t$. Since $K$ is hyperbolic, $t>0$, and also $t$ is even. We choose $\widehat{T}$ so that $t$ is minimal among all incompressible tori in $K(r)$. Let $T=\widehat{T} \cap E(K)$. Then it is a punctured torus properly embedded in $E(K)$ with $t$ boundary components, each having slope $r$ on $\partial E(K)$. Also $T$ is incompressible in $E(K)$ by the minimality of $t$.

We can isotope $T$ so that $S \cap T$ consists of loops and arcs. Since the cabling conjecture is true for genus one knots [2], $K(r)$ is irreducible. Then we can assume that no loop component of $S \cap T$ bounds a disk in $S$ or $T$ by the incompressibility of $S$ and $T$. Furthermore, we can assume that $\partial S$ intersects each boundary component of $T$ in $r$ points.

As usual ([4, 14]), the arc components of $S \cap T$ define a pair of graphs $G_{S}$ and $G_{T}$ on $\widehat{S}$, the capped-off surface of $S$, and $\widehat{T}$, respectively. Then $G_{S}$ has only one (fat) vertex with valency $r t$ and the arcs of $S \cap T$ give the edges. After labelling the boundary components of $T$ by $1,2, \ldots, t$ in the order in which they appear on $\partial E(K)$, the endpoints of edges in $G_{S}$ are labelled by $1,2, \ldots, t$, and this sequence is repeated $r$ times around the vertex of $G_{S}$. Similarly $G_{T}$ has $t$ vertices with valency $r$, but there is no label. We denote by $V_{i, i+1}$ the part of $V$ running from the component $i$ to $i+1$ of $\partial T$. (When $t=2, V=V_{1,2} \cup V_{2,1}$.)

Since $S$ and $T$ are boundary-incompressible, neither graph has trivial loops. The graph pair satisfies the parity rule [4]. It can be stated as follows in our setting: any edge of $G_{S}$ has the labels with distinct parities at its endpoints. In other words, any edge of $G_{T}$ connects two vertices with distinct parities.

In $G_{S}$, we can define a Scharlemann cycle, an extended Scharlemann cycle as in [14]. A (extended) Scharlemann cycle of length 2 is called an (extended) $S$-cycle for 
short. Also, a Scharlemann cycle with label pair $\{x, y\}$ is called a $(x, y)$-Scharlemann cycle. Finally, $\widehat{T}$ divides $K(r)$ into black and white sides, since $r>0$. Thus the disk faces of $G_{S}$ are divided into black faces and white faces, according to whether the faces lie in the black or white side of $\widehat{T}$. (Note that $G_{S}$ may have an annular face, which may contain essential loops of $S \cap T$.)

\subsection{Case $t \geq 4$.}

\section{LEMMA 2.3 .}

1. $G_{S}$ cannot contain an extended $S$-cycle.

2. $G_{S}$ cannot have more than $t / 2+2$ mutually parallel edges. If there is $t / 2+2$ mutually parallel edges, then the family contains two $S$-cycles with disjoint label pairs and $t \equiv 0(\bmod 4)$.

3. $G_{S}$ cannot contain three $S$-cycles with mutually disjoint label pairs.

Proof. (1) is Lemma 2.10 in [2]. (2) is Lemma 1.4 and Corollary 1.8 in [25]. (3) is Lemma 1.10 in [25].

Recall that the edges of $G_{S}$ are divided into at most three families of mutually parallel edges by [12, Lemma 5.1]. Let $A, B$ and $C$ be such three families, and let $|A|$ denote the number of edges in $A$, etc.

LEMMA 2.4. If $r$ is even, then $G_{S}$ cannot contain three mutually parallel edges.

Proof. Suppose that a family $A$, say, contains more than two edges. Since $|A|+$ $|B|+|C|=r t / 2$ is a multiple of $t$, the first and the last edge of $A$ have a common label (at different sides of $A$ ). If $|A|$ is odd, then the middle edge of $A$ has the same label on both endpoints, contradicting the parity rule. Thus $|A| \geq 4$, and hence $A$ contains an extended $S$-cycle, which is impossible by Lemma 2.3(1).

LEMMA 2.5. $r$ is odd.

Proof. Assume not. By Lemma 2.4, $r t / 2=|A|+|B|+|C| \leq 6$, and so $r \leq 3$. Thus $r=2$.

If $G_{S}$ contains only one family of mutually parallel edges, then it contains an extended $S$-cycle, a contradiction. If $G_{S}$ consists of two families $A$ and $B$, then $t=4$ and $|A|=|B|=2$ by Lemma 2.4 , since $|A|+|B|=t$. Then we may assume that $G_{S}$ contains a $(1,2) S$-cycle $\sigma_{1}$ and a $(3,4) S$-cycle $\sigma_{2}$. Since the edges of an $S$-cycle does not lie in a disk on $\widehat{T}$ [14, Lemma 3.1] (recall that $K(r)$ is irreducible), the edges of $\sigma_{i}$ form two disjoint essential loops on $\widehat{T}$ after shrinking the fat vertices into points. Let $f_{i}$ be the face of $\sigma_{i}$ for $i=1,2$. Shrinking $V_{1,2}$ to its core in $V_{1,2} \cup f_{1}$ gives a Möbius band $B_{1}$ such that $\partial B_{1}$ is the loop on $\widehat{T}$ formed by the edges of $\sigma_{1}$. Similarly we have another Möbius band $B_{2}$ from $V_{3,4} \cup f_{2}$. Since $\partial B_{1}$ and $\partial B_{2}$ bound an annulus $R$ on $\widehat{T}$, the union of $B_{1}, B_{2}$ and $R$ gives a Klein bottle in $K(r)$ meeting $V$ in two meridian disks. (See the proof of Lemma 3.10 in [14].) This implies that $E(K)$ contains a twice-punctured Klein bottle. But it yields a closed non-orientable surface in $E(K)$ by attaching an annulus in $\partial E(K)$ to it. This is absurd.

Hence $G_{S}$ consists of three families of mutually parallel edges. Since $t=|A|+$ $|B|+|C| \leq 6$, we have $t=4$ or 6 by Lemma 2.4 .

If $t=6$, then $|A|=|B|=|C|=2$. Then $G_{S}$ contains three $S$-cycles with mutually disjoint label pairs, which is impossible by Lemma 2.3(3). Hence $t=4$. We may assume that $|A|=|B|=1$ and $|C|=2$. Then all faces of $G_{S}$ are disks, and hence 
$S \cap T$ contains no loop component. But this configuration contradicts the black-white coloring of the faces. Thus the case $t=4$ is also impossible.

LEMMA 2.6. $G_{S}$ cannot contain more than $t / 2$ mutually parallel edges.

Proof. Assume $|A|>t / 2$. By Lemma $2.3(2),|A|=t / 2+1$ or $t / 2+2$. If $|A|=t / 2+1$, then the last edge of $A$ has the same label at both endpoints by the symmetry of labels. This is impossible by the parity rule. Hence $|A|=t / 2+2$, and so $t \equiv 0(\bmod 4)$ by Lemma $2.3(2)$. Then the first two edges and the last two edges of $A$ form $S$-cycles with disjoint label pairs. The edges of the two $S$-cycles form disjoint essential loops on $\widehat{T}$ after shrinking fat vertices into points. The torus $\widehat{T}$ is divided into two annuli $F_{1}, F_{2}$ by the two loops. The other $t-4$ fat vertices of $G_{T}$ make $(t-4) / 2$ pairs corresponding to the edges of $A$. Thus each annulus $F_{i}$ contains an even number of fat vertices in its interior. Then we have a Klein bottle in $K(r)$ meeting $V$ in an even number of meridian disks as in the proof of Lemma 2.5. This implies that $E(K)$ contains a Klein bottle punctured an even number of times. It yields a closed non-orientable surface in $E(K)$ by attaching suitable annuli on $\partial E(K)$, a contradiction.

PROPOSITION 2.7. If $t \geq 4$, then $r=1$.

Proof. By Lemma 2.6, $r t / 2=|A|+|B|+|C| \leq 3 t / 2$, and hence $r \leq 3$. Thus $r=1$ or 3 .

Suppose $r=3$. Then $G_{S}$ contains three families $A, B, C$ of mutually parallel edges, and $|A|=|B|=|C|=t / 2$. But $G_{S}$ contains an extended Scharlemann cycle of length 3 , which is impossible by [14, Theorem 3.2]. (Note that [14, p.610] uses the fact $K(r)$ does not contain a Klein bottle. But the arguments there work for our situation, because the fat vertices of $G_{T}$ make $t / 2$ pairs as in the proof of Lemma 2.6.) $\square$

2.2. Case $t=2$. Let $u_{1}$ and $u_{2}$ be the vertices of $G_{T}$. By the parity rule, each edge of $G_{T}$ connects different vertices $u_{1}$ and $u_{2}$. Then there are four edge classes in $G_{T}$, i.e., isotopy classes of edges of $G_{T}$ in $\widehat{T}$ rel $u_{1} \cup u_{2}$ (see [14, Figure 7.1]). We label an edge $e$ of $G_{S}$ by the class of the corresponding edge of $G_{T}$, and we call the label the edge class label of $e$.

LeMma 2.8. Two parallel edges in $G_{S}$ have distinct edge class labels.

Proof. If not, there are two edges which are parallel in both $G_{S}$ and $G_{T}$. Then $E(K)$ would be cabled [12, Lemma 2.1].

Proposition 2.9. If $r=4$, then $K$ is a twist knot and it bounds a once-punctured Klein bottle whose boundary slope is $r$.

Proof. Note that $G_{S}$ has exactly four edges. By the possibility of coloring of faces, there are two possible configurations for $G_{S}$ as shown in Figure 1 (after a homeomorphism of $S$ ). Here, the edges of the square are identified to form a torus in the usual way.

In the former case, the edges have mutually distinct edge class label by Lemma 2.8. Then the proof of Lemma 5.2 in [15] shows that $K(r)$ contains a Klein bottle. The conclusion follows from [22, Theorem 1.2].

In the latter case, we may assume that $G_{S}$ contains two black bigons. Unless these two black bigons have the same pair of edge class labels, $K(r)$ contains a Klein 

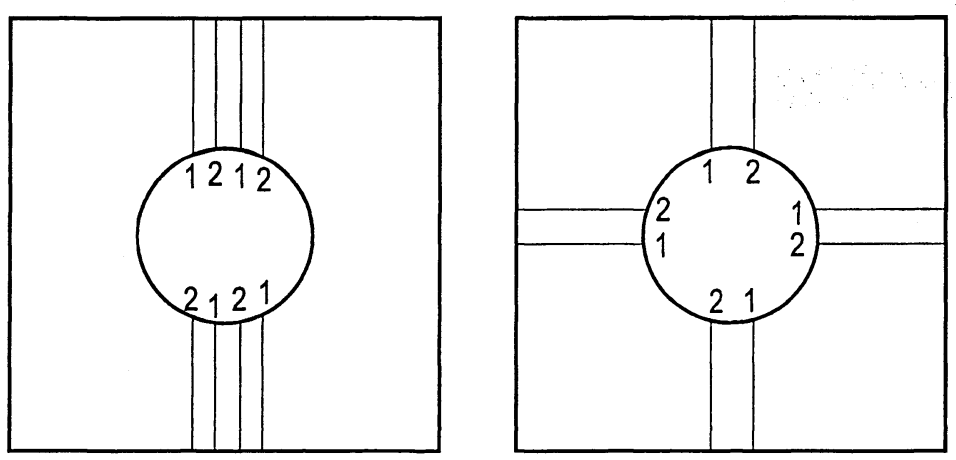

FIG. 1.

bottle again. Hence we suppose that the two black bigons have the same pair of edge class labels. Since all faces of $G_{S}$ are disks, $S \cap T$ contains no loop component.

Now $G_{S}$ contains a black $S$-cycle $\sigma_{1}$ and a white Scharlemann cycle $\sigma_{2}$ of length 4. Furthermore, all edges of these Scharlemann cycles lie in an essential annulus $R$ on $\widehat{T}$. Let $f_{i}$ be the face of $\sigma_{i}$ for $i=1,2$, and let $M=N\left(R \cup V \cup f_{1} \cup f_{2}\right)$. Then we can see that $M$ is not a solid torus by calculating its first homology group. Also it is easy to show that $R$ is essential in $M$, and both sides of $R$ are irreducible. Hence $M$ is irreducible, and so $\partial M$ is incompressible in $M$. Since it is disjoint from $V$, it is compressible in $K(r)$ by the minimality of $t$. Thus $\partial M$ is compressible in $E(K)$. It follows that $M^{\prime}=\operatorname{cl}(K(r)-M)$ is a solid torus. Therefore $M^{\prime} \cup N\left(f_{2}\right)$ is a handlebody of genus two, because we can regard $N\left(f_{2}\right)$ as a 1-handle attached to the solid torus $M^{\prime}$.

Let $\gamma$ be an arc on $f_{1}$ connecting the two arcs $f_{1} \cap V$. Since $N(\gamma)=N\left(f_{1}\right)$ and $N\left(R \cup V \cup f_{1}\right) \cong N\left(V \cup f_{1}\right), \operatorname{cl}(E(K)-N(\gamma)) \cong \operatorname{cl}\left(K(r)-N\left(V \cup f_{1}\right)\right) \cong M^{\prime} \cup N\left(f_{2}\right)$. Thus $K$ has an unknotting tunnel $\gamma$ which lies in $S$. Then $K$ is 2-bridge [11, Lemma 6.1]. According to [3], genus one 2-bridge knots with toroidal slope 4 are twist knots, and 4 is the boundary slope of a once-punctured Klein bottle bounded by such a knot. This completes the proof. $\square$

LEMma 2.10. Assume $r \neq 4$. Then $G_{S}$ cannot have more than three mutually parallel edges.

Proof. Suppose that there are four mutually parallel edges. Then there are two bigons with the same color among these edges. If these bigons have distinct pairs of edge class labels, then $K(r)$ contains a Klein bottle as before and $r=4$ by [22]. Thus the two bigons have the same pair of edge class labels. This implies that there are two parallel edges with the same edge class label, which contradicts Lemma 2.8. $\mathrm{B}$

Let $F$ be a family of mutually parallel edges in $G_{S}$.

LEMma 2.11. Assume $r$ is odd. If $F$ is not empty, then $|F|=1$ or 3 .

Proof. By Lemma 2.10, $|F| \leq 3$. If $|F|=2$, then each edge of $F$ has the same label at its endpoints by the symmetry of labels. This is impossible by the parity rule. 口

LEMMA 2.12. $r=3$ is impossible. 

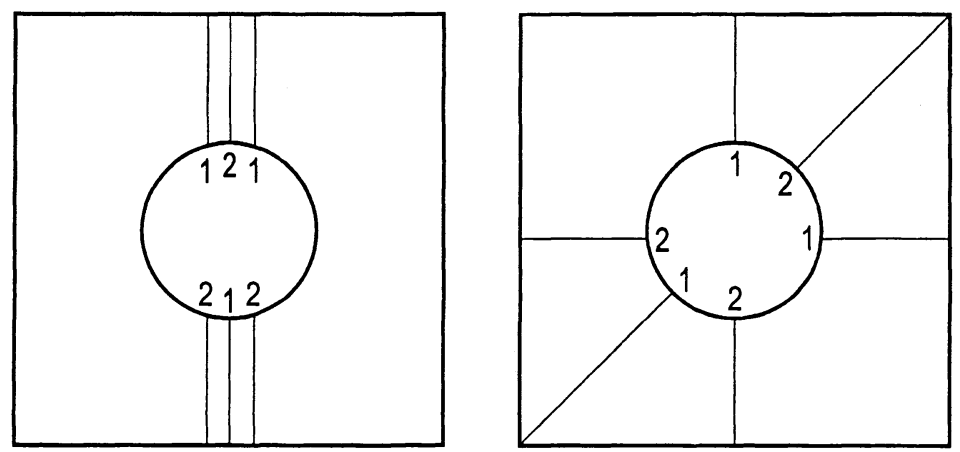

FIG. 2.

Proof. Note that $G_{S}$ has just 3 edges. By Lemma 2.11 and the possibility of coloring of faces, there are only two possibilities for $G_{S}$ as shown in Figure 2.

For the first case of Figure 2, $S \cap T$ contains loops, because of the coloring of the faces of $G_{S}$. Recall that such a loop does not bound a disk in either $S$ or $T$. On the other hand, the edges have mutually distinct edge class labels by Lemma 2.8. Hence any loop of $S \cap T$ bounds a disk in $T$, a contradiction.

For the second case, $G_{S}$ contains a black Scharlemann cycle $\tau_{1}$ and a white Scharlemann cycle $\tau_{2}$, both of length 3 . The edges of $\tau_{1}$ (and hence $\tau_{2}$ ) lie in an essential annulus $R$ on $\widehat{T}$ ([14, Lemma 3.7]). Let $f_{i}$ be the face of $\tau_{i}$, and let $M=N\left(R \cup V \cup f_{1} \cup f_{2}\right)$. We may assume that $R$ divides $M$ into two pieces $M_{1}$ and $M_{2}$, where $f_{i} \subset M_{i}$. By [14, Lemma 3.7], $M_{i}$ is a solid torus and the core of $R$ runs three times along the core of $M_{i}$ for $i=1,2$. Then $M$ is a Seifert fibered manifold over the disk with two exceptional fibers of index 3 . In particular, $\partial M$ is incompressible in $M$. Then, as in the proof of Proposition 2.9, the complement $M^{\prime}=\operatorname{cl}(K(r)-M)$ is a solid torus. Hence $K(r)$ is a Seifert fibered manifold over the 2-sphere with at most three exceptional fibers. (Recall that $K(r)$ is irreducible.) But this contradicts that $K(r)$ is toroidal.

LEMMA 2.13. $r=5$ is impossible.

Proof. By Lemmas 2.11 and the possibility of coloring of faces, there is only one possibility for $G_{S}$ as shown in Figure 3. Since $r$ is integral, the points of $\partial S \cap u_{i}$ appear successively on both $\partial S$ and $u_{i}$ for $i=1,2$. By using this observation, we can determine $G_{T}$ as shown in Figure 3. Then $G_{S}$ contains a black $S$-cycle $\sigma$ and a white Scharlemann cycle $\tau$ of length 3 , such that the edges of $\sigma$ and $\tau$ are contained in the same essential annulus on $T$. Then the argument in the proof of Lemma 2.12 works.

Proof. [Proof of Theorem 1.2] By Lemmas 2.1, 2.2, 2.12 and 2.13, and Proposition 2.7, we have $|r|=0,1,2$ or 4 . Also, if $|r|=2$ or 4 , then $t=2$ by Proposition 2.7. Finally, if $|r|=4$, then we have the desired conclusion by Proposition 2.9.

3. Alternating case. In this section, we prove Theorem 1.3. Let $K$ be an alternating hyperbolic knot, and let $r$ be a toroidal slope.

Proof. [Proof of Theorem 1.3] By Lemmas 3.1 and 3.3 of [1], $r$ is an integer divisible by 4 , and $K$ is either a 2 -bridge knot or a pretzel knot of type $\left(1 / q_{1}, 1 / q_{2}, 1 / q_{3}\right)$, 

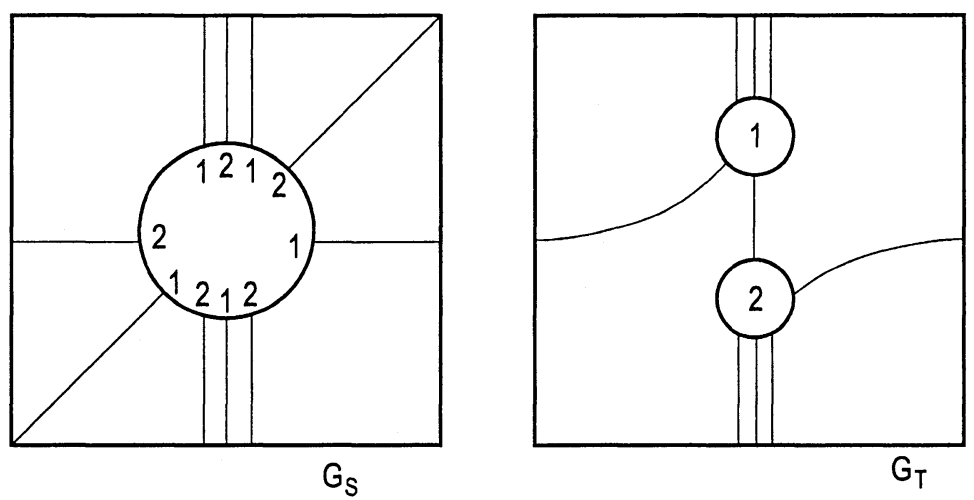

FIG. 3.

where all $q_{i} \geq 2$. (See also [19].) When $K$ is 2-bridge, [3] shows that $K$ is either genus one or of the form $\left[b_{1}, b_{2}\right]$ with $\left|b_{1}\right|,\left|b_{2}\right|>2$. In either case, we see that $|r| \leq 4 g(K)$, and that if $r=4 g(K)$ then $K$ bounds a once-punctured Klein bottle.

Next assume that $K$ is a pretzel knot of type $\left(1 / q_{1}, 1 / q_{2}, 1 / q_{3}\right)$. If all $q_{i}$ are odd, then $K$ has genus one, and 0 is the only toroidal slope [1, Lemma 3.3]. If one of $q_{i}$, $q_{1}$ say, is even, then $K$ bounds a once-punctured Klein bottle whose boundary slope is $2\left(q_{2}+q_{3}\right)$, as a checkerboard surface in the standard diagram. Let $s=2\left(q_{2}+q_{3}\right)$. By $[9,10], K$ has genus $s / 4$, that is, $s=4 g(K)$.

Claim 3.1. $K(s)$ is toroidal.

Proof. [Proof of Claim 3.1] Since $K(s)$ contains a Klein bottle, there are three possibilities for $K(s)$ : toroidal, reducible, or a Seifert fibered manifold with finite fundamental group (more precisely, a prism manifold). First, $K(s)$ is irreducible, because $K$ is strongly invertible ([7]). Also non-trivial surgery on an alternating knot yields a manifold with infinite fundamental group [5]. Therefore $K(s)$ must be toroidal.

Since $s$ is the only toroidal slope of $K[1$, Lemma 3.3], this completes the proof of Theorem 1.3.

There are examples of alternating hyperbolic knots showing that the estimate of Theorem 1.3 is best possible for each genus. In case of genus one, twist knots give such examples. For $g \geq 2$, the 2-bridge knot $\left[b_{1}, 2 g\right]\left(b_{1} \geq 3\right.$, odd) has genus $g$ and a toroidal slope $4 g([3])$. Also it bounds a once-punctured Klein bottle whose boundary slope is $4 g$.

\section{REFERENCES}

[1] S. BOYER AND X. ZhANG, Cyclic surgery and boundary slopes, in Geometric topology (Athens, GA, 1993), pp. 62-79, AMS/IP Stud. Adv. Math., 2.1, Amer. Math. Soc., Providence, RI, 1997.

[2] S. Boyer AND X. ZhANG, Reducing Dehn fillings and toroidal Dehn fillings, Topology Appl., 68 (1996), pp. 285-303.

[3] M. BRITtenham AND Y. Q. WU, The classification of exceptional surgeries on 2-bridge knots, Comm. Anal. Geom., 9 (2001), pp. 97-113. 
[4] M. Culler, C. MCA. Gordon, J. Luecke and P. Shalen, Dehn surgery on knots, Ann. of Math., 125 (1987), pp. 237-300.

[5] C. Delman AND R. ROBERTS, Alternating knots satisfy strong property $P$, Comment. Math. Helv., 74 (1999), pp. 376-397.

[6] N. Dunfield, A table of boundary slopes of Montesinos knots, Topology, 40 (2000), pp. 309-315.

[7] M. EUdAVE-MUÑoz, Band sums of links which yield composite links. The cabling conjecture for strongly invertible knots, Trans. Amer. Math. Soc., 330 (1992), pp. 463-501.

[8] D. GABAI, Foliations and the topology of 3-manifolds, III, J. Differential Geom., 26 (1987), pp. 479-536.

[9] D. GABAI, The Murasugi sum is a natural geometric operation, In Low-dimensional topology (San Francisco, Calif., 1981), pp. 131-143, Contemp. Math., 20, Amer. Math. Soc., Providence, RI, 1983.

[10] D. GABAI, Genera of the arborescent links, Mem. Amer. Math. Soc., 59 (1986), pp. 1-98.

[11] H. GodA AND M. TERAGaITo, Dehn surgeries on knots which yield lens spaces and genera of knots, Math. Proc. Cambridge Philos. Soc., 129 (2000), pp. 501-515.

[12] C. MCA. GoRdon, Boundary slopes of punctured tori in 3-manifolds, Trans. Amer. Math. Soc., 350 (1998), pp. 1713-1790.

[13] C. McA. Gordon, Dehn filling: a survey, Knot theory (Warsaw, 1995), pp. 129-144, Banach Center Publ., 42, Polish Acad. Sci., Warsaw, 1998.

[14] C. MCA. GoRdon AND J. LUECKE, Dehn surgeries on knots creating essential tori, I, Comm. Anal. Geom., 3 (1995), pp. 597-644.

[15] C. MCA. Gordon AND J. LuECKE, Toroidal and boundary-reducing Dehn fillings, Topology Appl., 93 (1999), pp. 77-90.

[16] C. MCA. Gordon AND J. LuECKE, a private communication.

[17] A. Hatcher and U. Oertel, Boundary slopes for Montesinos knots, Topology, 28، (1989), pp. $453-480$.

[18] K. IchihARA, Exceptional surgeries and genera of knots, Proc. Japan Acad. Ser. A, 77 (2001), pp. 66-67.

[19] R. PATtoN, Incompressible punctured tori in the complements of alternating knots, Math. Ann., 301 (1995), pp. 1-22.

[20] D. Rolfsen, Knots and links, Mathematics Lecture Series, 7, Publish or Perish, Inc., Berkeley, Calif., 1976.

[21] M. SCHARLEMANN, There are no unexpected tunnel number one knots of genus one, preprint.

[22] M. Teragaito, Creating Klein bottles by surgery on knots, J. Knot Theory Ramifications, 10 (2001), pp. 781-794.

[23] M. Teragaito, Toroidal surgeries on hyperbolic knots, Proc. Amer. Math. Soc., 130 (2002), pp. 2803-2808.

[24] W. Thurston, The geometry and topology of 3-manifolds, Princeton University, 1978.

[25] Y. Q. Wu, Dehn fillings producing reducible manifolds and toroidal manifolds, Topology, 37 (1998), pp. 95-108. 